\title{
EL SOL COMO FUNDAMENTO CURATIVO DE LAS TERAPIAS MAYAS YUCATECAS EN EL PERÍODO COLONIAL
}

\author{
MónICA Chávez GuZMáN \\ DGEP, UNAM ${ }^{1}$
}

En este artículo se aborda uno de los aspectos fundamentales para poder entender la concepción maya yucateca sobre la recuperación de la salud del enfermo: los poderosos dones "calientes" de los remedios medicinales, particularmente de las plantas, reflejados en varios documentos coloniales.

Dentro de los textos analizados se encuentran los diccionarios Calepino de Motul, el Bocabulario de Maya Than, el Ritual de los Bacabes, el Libro de los Cantares de Dzitbalche, así como los libros Chilam Balames de Nah, Kaua, Ixil, Maní y Tekax. Todos ellos son textos provenientes de la península de Yucatán, elaborados por indígenas y españoles, en maya y español.

Estos manuscritos presentan una mezcla cultural maya-europea con un frecuente ajuste de las ideas de los conquistadores a la lengua nativa, principalmente los Chilam Balames. En estos últimos se observa cierta libertad de interpretación de las ideas españolas por parte de los escribanos yucatecos que plasmaron la información y, a veces, hasta parece haberse complementado el pensamiento extranjero con las opiniones de los indígenas, armonizando las ideas propias con las nuevas aportaciones. Al analizar las referencias en lengua maya desde el punto de vista semántico y etimológico en los textos, se obtienen interesantes datos sobre la percepción y concepción de la enfermedad, y acerca de los tratamientos de sanación originados a partir del pensamiento cosmogónico indígena, así como la explicación del funcionamiento de las terapias.

\section{El "calor" solar de las plantas medicinales y del ser humano}

Iniciaremos con la manera como se expresa la capacidad de curar de las plantas, de brindar sus dones, a través del vocablo kinam, ${ }^{2}$ en la expresión kinam

\footnotetext{
${ }^{1}$ Algunos datos del presente artículo son parte de la tesis de doctorado en Estudios Mesoamericanos que estoy llevando a cabo.

${ }^{2}$ La escritura de las palabras mayas que se ofrece es como aparece en los documentos.
} 
xiuoob, 'virtud de las yerbas' (Ciudad Real, 1995, I: 37). Kinam significa "fuerza", "reciura", "fortaleza", y es un término clave para entender cómo actúan los vegetales al ser aplicados al ser humano, además del concepto de "frío-calor" en la enfermedad. Este vocablo viene de la raíz kin, 'sol', y muchas de las palabras derivadas de ella hablan de "calidez". Kinam no es solamente la virtud que poseen las plantas, sino también las piedras y los animales (ibid. I: 424, 425 y 734), pero en este artículo se hace énfasis en las plantas, porque en los documentos coloniales sobresalen notablemente de entre los otros elementos curativos de la naturaleza. Los manuscritos del siglo xvı más cercanos al período de la conquista, al igual que el Ritual de los Bacabes, texto redactado alrededor de 1779 , cuyo contenido parece ser una copia de otro elaborado por lo menos en la segunda mitad del siglo xv, muestran escasa presencia de fauna y minerales y son muy abundantes en referencias sobre flora medicinal.

En los textos coloniales, kinam también se relaciona con la fortaleza de una persona o animal que inspira temor, respeto, veneración o ferocidad (ibid. 1: 425). Recordemos que de acuerdo con mayas yucatecos de mediados del siglo xx, kinam era la fuerza irradiada por algunas personas, animales o piedras, que afectaba la conducta o el estado del organismo de quienes se encontraban cerca de ellos, y era un mal en el cuerpo causante de enfermedades (Villa Rojas, 1981: 22-23). Los tzotziles consideraban que los que contactaban con frecuencia a las deidades y a los viejos acumulaban "calor" en sus corazones y causaban temor, pues su poder dañaba a los que no tenían suficiente vigor para resistir sus emanaciones (Guiteras, 1965: 248).

El "calor" solar del individuo (kin) en los mayas coloniales, que podía llegar a ser poderosamente fuerte (kinam), coincide en algunos aspectos con el tonalli de los antiguos nahuas, pues también se refiere al "calor" del astro rey; comparación que no pretende establecer paralelos exactos, pues no los hay, pero que muestra una vez más la gran influencia que existió entre ambas culturas.

El tonalli actuaba como control de la temperatura del hombre, lo tenían todos los seres vivos, los objetos, se acumulaba conforme envejecía, era un elemento muy importante en el funcionamiento del organismo, condicionante de la salud, generador de enfermedades, y se decía que al asustarse una persona la abandonaba su tonalli (López Austin, 1996, I: 230, 233, 236-237, 288, 289 y 401.

Kinam parece haber sido el "calor" y la "fuerza" que poseían los líderes y los sabios, como una "emanación" virtuosa alimentada por el sol, incrementada con los años, que llegaba a tener tal magnitud que afectaba a los débiles. Lo anterior parece confirmarse en la descripción en español de los documentos coloniales, en los que se encuentra registrado ah kinam, quien es una persona que causa espanto, temor y reverencia; al igual que ah kinam than, líder importante por sus palabras, a quien "nadie le osa hablar por miedo o respeto" (Ciudad Real, op. cit., I: 29).

Al parecer, el hombre común, no anciano, poseía un "calor" solar moderado fundamental para poder vivir, y al igual que el tonalli náhuatl, podía desequili- 
brarse ocasionando que se padecieran males "fríos" o excesivamente "calientes", como veremos más adelante.

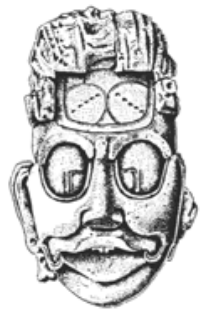

Figura 1. Dios sol.. Cabeza de estuco con los dientes limados típicamente y una enroscadura en cada comisura de la boca. Palacio de Palenque.

Tomado de Thompson, 1975: 291

La ceremonia zihil, 'nacer de nuevo', que Landa llamó bautismo en la Relación de las cosas de Yucatán, por su vinculación con el agua, se realizaba en niños y muchachas púberes con el fin de que en su futuro tuvieran un buen comportamiento y las deidades los protegieran de ser atacados por entes malignos. En las mujeres era además un rito que les brindaba licencia para poder casarse:

A las niñas poníanles como madrina a una mujer anciana y a los niños un hombre que los tuviese a su cargo [...] Los chaces iban luego a los niños y ponían a todos en las cabezas, sendos paños blancos que sus madres traían para ello [...] levantábase el principal que los padres de los muchachos habían elegido para esta fiesta y con un hueso que el sacerdote le daba iba a los muchachos y amagaba a cada uno nueve veces en la frente; después, mojábanle con el agua de un vaso que llevaba en la mano y untábales la frente y las facciones, y entre los dedos de los pies, y de las manos, sin hablar palabra [...]. Acabada esta untura se levantaba el sacerdote y les quitaba los paños blancos de la cabeza y otros que tenían colgados a las espaldas en que cada uno traía atadas unas pocas plumas de unos pájaros muy hermosos y algunos cacaos todo lo cual recogía uno de los chaces, y luego el sacerdote cortaba a los niños, con una navaja de piedra, la cuenta que 
habían traído pegada en la cabeza; tras de esto iban los demás ayudantes del sacerdote con un manojo de flores y un humazo que los indios usan chupar y amagaban con cada uno de ellos nueve veces a cada muchacho y después dábanles a oler las flores y a chupar humazo ( Landa, 1986: 44). ${ }^{3}$

Como se podrá observar, esta ceremonia implicaba en ciertos momentos algo similar a la atracción del tonalli náhuatl que gustaba del agua y el cacao, y para su mantenimiento en la persona requería de acciones preventivas dirigidas principalmente a la mollera, la coronilla, lugar de salida del mismo, así como a la frente, el rostro, el cabello, o la cabeza en general. También entre los tzotziles actuales la "sombra", una de sus "fuerzas vitales", que algunos investigadores ligan con el tonalli, y que puede perderse, es atraída o recuperada por aquello que éste apetece, que además del agua, incluye elementos aromáticos, como las flores, el copal y el tabaco (López Austin, op. cit.: 224, 234 y 251). Como vimos, empleados también en la ceremonia zihil.

Entre los nahuas esta ceremonia se realizaba cuando se sacaba al niño por primera vez a que recibiera los rayos del sol, para que el astro rey le imprimiera de manera definitiva una "energía" favorable o desfavorable a través de su contacto. De esta manera se determinaba para siempre la tendencia del individuo en cuanto a su personalidad, oficios, debilidades y virtudes de acuerdo con el calendario, ya que el "calor" o luz llegaba a la tierra con diferente influencia de manera diaria. El calendario náhuatl se sustentaba en el tonalli, cuya palabra viene de tona, que significa además de irradiación calorífica, signo del día, destino y propiedad de la persona (ibid.: 230-232). El que este calendario y el maya compartieran sus bases, aunque le proporcionaran nombres diferentes (Aveni, 1993: 230-232) y tuvieran adaptaciones particulares, hace pensar en la posibilidad de que el segundo también hubiera fundamentado el suyo en el influjo solar.

\section{Enfermedades "frías" y "calientes" aliviadas con el "calor" del astro rey}

Se ha tratado ya en numerosas publicaciones un tema tan controvertido como el del origen del concepto frío-calor en América. ${ }^{4}$ Desde tiempos antiguos hasta la actualidad, los hombres de diversas partes del mundo han coincidido en atribuir características "frías" o "calientes" a los elementos de la tierra, entre ellos, personas, plantas, alimentos, medicinas y enfermedades. Estas calificaciones no siempre se refieren a su temperatura, en tanto calor de los cuerpos

\footnotetext{
${ }^{3}$ Para los nahuas antiguos, el tonalli se alojaba en la cabeza y éste podía ser creado en alguno de los nueve pisos más altos del cielo, quizá por eso el ademán de tocar la frente nueve veces (López Austin, op. cit:: 224, 227, 232, 234 y 236).

${ }^{4}$ Para ampliar sobre el tema se pueden consultar, entre otros textos, a Foster, 1994 y López Austin, 1996.
} 
como cualidad "física", sino a una especie de "energía" o propiedad de los seres vivos y no vivos, difícil de describir.

Los españoles conquistadores tenían también esta creencia, sustentada en la Teoría de los humores, en la cual, para poder aliviar un padecimiento "frío", era necesario aplicar remedios "calientes", y contra los males "calientes", remedios "fríos". Es decir, era imprescindible curar al enfermo con tratamientos de cualidades contrarias. ${ }^{5}$

En los documentos mayas de los siglos xvi al xvIII, el exceso de "calor"6 adquirido de manera brusca se manifiesta a través de chacau, 'calentura' y kaak, 'fuego', incluido en varias enfermedades graves de la piel, como uech kaak, 'sarna o granos de fuego con mucha comezón' y chuchum kaak, 'pezón de fuego o incordios', similares a tumores grandes con pus. Los males "fríos" incluyen en sus nombres los términos ziz, 'frío o fresco'; ziizkab, 'parálisis, adormecimiento e hinchazón' y zizthanen, 'fuerza o poder fríos'. También zac, que significa 'blanco e imperfecto', parece asociado al "frío" en ciertos padecimientos, como zackuxnak, 'panza blanca que duele', descrita como diarrea fría; ppoc zacan, 'sombrero o caperuza o sombrero blanco'; la hinchazón con dolor y el "frío" en las rodillas por caminar mucho, como una especie de gota; desmayos [zac cimil, "muerte blanca o imperfecta'| y males de los ojos como zac, 'catarata', al parecer, por su clara asociación con la formación blanquecina del ojo. Inclusive hay ciertas partes del cuerpo relacionadas con el "frío", como ceel ich, 'lo frío del ojo', 'lo blanco', reconocido también como zacmapil ich (Ciudad Real, op. cit.: 115, 151-152, 154-155, $169,170,224,250,402,667$ y 756). Es de notar que al analizar la información anterior pareciera que en algunos casos, como la parálisis, el adormecimiento y la hinchazón, se refirieran a secciones del cuerpo en donde el "calor" solar del individuo no fluyera de manera normal o se detuviera.

En la traducción al español de los redactores de los textos coloniales se presentan algunos otros padecimientos que involucran el "frío" o el "calor", pero no se puede confirmar a través del significado de las palabras en maya, por lo que no se descarta que se trate de aportaciones de la cultura médica humoral de los escribanos ibéricos. Por ejemplo, thun, 'venda o trenzadera', se describe en castellano como diarrea ocasionada por frialdad con pujos, al igual que kulim can, 'chinche recia', "una especie de enfermedad como fuego" (ibid.: 437 y 740).

La idea de las enfermedades "frías" o "calientes" que se curan con plantas de cualidades contrarias al modo europeo, no se manifiesta en los textos colo-

\footnotetext{
${ }^{s}$ Esta teoría señalaba como propiedades del cuerpo, el calor, el frío, la sequedad y la humedad, originadas a partir de los elementos básicos del universo: aire, agua, tierra y fuego. En el organismo se concebían cuatro humores: bilis, flema, sangre y pituita. La bilis amarilla se consideraba sobre todo caliente y seca; la sangre, caliente y húmeda; la bilis negra, seca y fría, mientras que la humedad y la frialdad eran constituyentes prioritarios de la pituita o flema. La salud dependía de la correcta composición y equilibrio entre estos componentes (García, 1972: 94, 235-236 y 252).

${ }^{6}$ No acumulado de manera paulatina con el transcurrir del tiempo, o con el contacto frecuente con las deidades a través de los rituales.
} 
niales tempranos, del siglo xvı, sino más bien se trata de yerbas medicinales con dones cálidos solares como virtud del astro rey para aliviar males tanto "fríos", como "calientes". Es decir, pese a que se observan ciertas coincidencias entre los conceptos indígenas y españoles con respecto a la enfermedad, también se presentan discrepancias importantes en lo concerniente a la solución dicotómica del mal. Solamente se hace referencia a una planta, al parecer introducida por los europeos de acuerdo con su nombre (Sosa, et al., 1985: 48), con una posible connotación de frialdad en su traducción, y se le llama zizim, ${ }^{7}$ 'ajenjos verdes de esta tierra' (Ciudad Real, op. cit.:169), pero el escribano español no lo especifica en su descripción en castellano, y ni siquiera le otorga alguna propiedad medicinal.

En la "Relación de la Ciudad de Mérida", al igual que en otras incluidas en las Relaciones histórico-geográficas de la Gobernación de Yucatán, se manifiesta la diferencia en los conceptos sobre "frío-calor" entre los ibéricos y mayas, pues a pesar de que en ella se señala el conocimiento indígena de clasificación "fríocaliente" de las plantas, el encomendero que hace esta observación reconoce un entendimiento diferente del suyo, y señala la ignorancia de los nativos hacia sus propiedades. Por lo tanto, limitaciones de la sabiduría local para aprovecharlas:

Hay en esta tierra mucha cantidad de hierbas medicinales de diferentes propiedades, y si hubiese persona en ella que tuviese conocimiento de ellas las hallaría de grandísima utilidad y efecto, porque los indios naturales no hay enfermedad a que no apliquen hierbas, pero preguntándoles razón de su propiedad no saben dar otra más de ser fría o caliente, y que suelen usar de ellas para aquél efecto que las aplican (Relaciones histórico-geográficas de la Gobernación de Yucatán, 1983, l: 78).

Según parece, el español se refería a la falta de conocimiento de los indígenas sobre la Teoría de los humores, sus explicaciones sobre la salud, la enfermedad, los métodos curativos, y es probable que los mayas entrevistados no hubieran querido comentar la concepción pagana de "calor"-solar-virtud (kinam), mencionada anteriormente.

En el Códice Dresde, anterior a la conquista, elaborado aproximadamente entre 1200-1250 d.C., la imagen de Ixchel, diosa de la luna, la enfermedad y la medicina, que representa el envío de sus designios o suerte a los seres humanos, ${ }^{8}$ es acompañada de los símbolos chac y sac. El primero, como significado de lo grande, rojo, implica la enfermedad grave, como una gran peste o una gran mortandad. Sac podría corresponder al color blanco de la luna, que igual-

\footnotetext{
${ }^{7}$ Artemisia vulgaris. Ziz, 'cosa fría o fresca' (Ciudad Real, op. cit., I: 169).

${ }^{s}$ Que merecería, al igual que el sol, un espacio especial para presentar su influencia dual tanto en la salud, como en la pérdida de la misma.
} 
mente es la raíz de 'tejer', actividad representativa de la diosa (Thompson, 1993: 42, 128-129). Pero por lo señalado líneas arriba, quizá chac también se relacionaba con los padecimientos con "calor" excesivo, como aquellos que involucran kaak, fuego, símbolo que también acompaña a la diosa lunar en varias de las representaciones de dicho códice (ibid.: 125), como las mortales viruelas (metnalil kaak, 'fuego del Mitnal') y el sarampión (uzan kaak, 'salpullido de fuego'). En contraste, sac, 'blanco', en la acostumbrada percepción maya dual del cosmos, quizá se refiriera también a los males "fríos", pues a Ixchel no sólo se le asociaba con este color, sino con todos los colores, pues era la del "arco del cielo", del arco iris (Barrera M. et al., 1976: 499).

En textos posteriores al siglo xvi, en maya, como los Chilam Balames, encontramos escasos males que se alivian con contrarios, es decir, "frío" para "calor" y viceversa, pero en la gran mayoría se recomiendan terapias calientes para todos los padecimientos. Por ejemplo, en el Chilam Balam de Kaua en las secciones en las que se habla del alivio para varios tipos de fiebre (chacau), como la persistente que aparece cada vez que anochece, se recomienda hervir en agua la fragante planta de la noche (ákab xiuil) y aplicarla en baño tan caliente como el paciente soporte cada mañana temprano, de preferencia cuando el sol esté suficientemente cálido (ti choco hi bahun uchac u mukik kohane, lay licil y utzcinicob amal haascab chacau kin dzedzec u dzacal chocouil licil u yanhal ti akab sansamal ti uinic). Además se enfatizan sus propiedades al señalar que esta planta cura por su jugo caliente (tu men licil u toxicob u chocou haail xiu lae) (Bricker y Miram, 2002: 328-330).

La virtud otorgada a las yerbas medicinales por el astro rey también se menciona en dos versiones de los conocidos como "Libros del judío", en español, señaladas por Barrera Vásquez como posibles copias de recetarios de los siglos $\mathrm{xvl-xvII}$, que recopilan remedios típicos, tanto de la terapéutica europea, como indígena; pues al describir las propiedades de la Artemisa se menciona que esta planta tiene la cualidad de curar muchas enfermedades y "estas virtudes las recibe de una estrella que los astrónomos llaman sol" (Ossado, 1983: 1; 1992: 589).

\section{La lucha del ser humano por mantener su "calor" solar}

En el análisis de todos los textos coloniales consultados se observa que hay una constante y permanente lucha entre los hombres por mantener su "calor", y los que carecen de cuerpo y vagan por el cosmos, seres "fríos", como los muertos o los aires ( $i k$ ) personificados, que tratan de arrebatárselo. Por ejemplo, en el Chilam Balam de Maní (Pérez, 1949: 59-61) y en el Kaua, se señala que en el cielo habitan fuerzas malignas que en algunas ocasiones, como la entrada de la constelación de Leo, bajan y se esparcen en la tierra. Pueden llegar a través de la neblina, el rocío, y traen consigo padecimientos a las personas, les ocasionan "frío" (figura 2): 
Lay sac kuyuch muyal

yan t u nak can lae.

Ti yan ah chibalob. ${ }^{9}$

Bai u cah cane.

Ah chibal canob

Ba ix ma ah chibal caan xani

Yan uai y okol cabil lae

Y u hach lobil sisob

Celob u naob ti can

ti c u ch'a kinam tu lacal

iHe $\mathrm{t} u$ lubul $\mathrm{u}$ yahil ti uine.

Hach ma chan dzaci

Amal y ocol kin $u$ chucsah ti uinic [...]

[...] Ca yantal ton sis amal y ocol

kin $[\ldots]$

[...] Lai licil u tsayal hauai y sook

bai bacab sise [...]

[...] Bai t uy ahal caab

lic ix u lubul chac yeeb

chac onob can

sis u cuch lae

lai...saac kuyuch lae [...]
Ésta blanca nube de la capa del cielo que se encuentra en un lugar aparte. Allá está "el del dolor escociente". Esa es su propiedad, su naturaleza. "El del dolor escociente" que muerde. Aun sin "el del dolor mordiente" del cielo también es su propiedad [el que existan] compartimientos encima de la tierra |en el cielo...] muy malos y "fríos".

Frías son sus casas en el cielo. iAy! el cobro [Lgoteo?] del dolor por todos lados.

Veis aquí el descenso del mal en los hombres.

No es pequeña su cura.

En cada puesta del sol [se presenta] el asimiento del hombre [del mal...]

Existe una bolsa de "frío" en cada atardecer[...].

[...] Por ello se pega la lepra y la sarna de acuerdo al Bacab del "frío" [...].

Se siente al amanecer.

Así pasa con el descenso de la neblina, del rocío, del sereno, que son fuertes, graves[...].

Fría es la carga de este...blanco apartado[...].

(Bricker y Miram, op. cit.: 97 y 99)

El atardecer, cuando iban desapareciendo los rayos solares y se anunciaba la noche, era sumamente peligroso, pues daba origen a "la maldad de la creación, a la maldad de la noche" (Ritual de los Bacabes, 1987: 308), ya que la oscuridad

\footnotetext{
${ }^{9}$ Ah chi'bal, 'el que muerde o pica'. También podría hablar de ch'ibal, 'casta, linaje patrilineal' (Barrera Vásquez, et al., pp. 92,133-134; Ciudad Real, Calepino de Motul, R. Arzápalo, (ed.), pp. 20 y 262; Bocabulario de Maya Than, R. Acuña (ed.), p. 367), para referirse a los antepasados, a los muertos fríos que llegan del inframundo a la tierra, a través de la ceiba del centro del cosmos y el cielo, para robarles su "calor" a los vivos, como se explica en la siguiente figura. En este caso, el atacante es el ser que muerde o pica.
} 


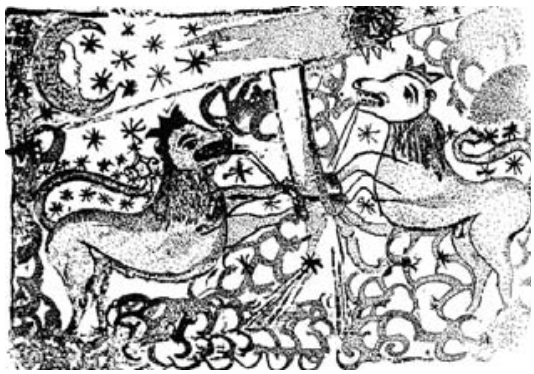

Figura 2. Ilustración del. Chilam Balam de Kalla (Bricker y Miram, op. cit.: 98).

Posiblemente muestra la creencia que mantienen algunos mayas actuales, en la ceiba del centro del cosmos (eje central del dibujo) como vía de comunicación y acceso de los antepasados que habitan el inframundo, con el cielo y la tierra (Thompson, op. cit: 243). Los muertos, seres "fríos", bajan ${ }^{\text {to }}$ con la neblina y el rocío, principalmente en ciertas constelaciones, como la que recibe el nombre de Leo en el zodiaco español. Influjo que parece ser representado por el artista mediante el contacto de los Leones con el eje cósmico. ${ }^{11}$ Conceptos que se manifiestan de manera frecuente en el Ritual de los Bacabes.

hacía más susceptibles a los hombres de ser atacados por los seres sedientos de su "energía". También la conclusión del año parecía facilitar la pérdida del "calor" vital en el individuo, por eso se consideraba un período aciago y eran

${ }^{10}$ Entre los ixiles de Guatemala, toda enfermedad es causada por el movimiento en el espacio de seres que salen del lugar que les corresponde, y las fiebres, espasmos o calambres son causados por las almas ancestrales (B. y L. Colby, 1986: 246).

${ }^{11}$ La ilustración es una muestra de la adaptación de los conceptos españoles al pensamiento maya, tan característica de los libros Chilam Balam, pues el dibujo y el texto forman parte de la explicación de los signos del zodiaco europeo, complementada con ideas que no coinciden con las de los conquistadores. En el escrito se le nombra a la constelación como Cáncer, que antecede a Leo, en una posible adecuación del escribano indígena, como sucede en otra parte del Chilam Balam de Kaua, que Bricker y Miram explican como una adaptación de Cáncer a Géminis, con la figura de pájaro que conocían los mayas, pues a pesar de que se decía que Cáncer caía el 12 de junio en tiempos coloniales, como lo dice el Kaua, la constelación que aparecía en esas fechas eran los gemelos. Para más información se recomienda leer: An encounter of two worlds, The book of Chilam Balam of Kaua (Bricker y Miram, op. cit.: 95). 
días de guardar. Los indígenas solían "salir muy poco de casa estos cinco días [...] no se peinaban, ni lavaban, ni las mujeres ni los hombres se espulgaban, ni hacían cosa servil o trabajosa, porque temían que les sucediera ningún mal si lo hacían" (Landa, 1986: 88). ${ }^{12}$ Pasado este período empezaba el año nuevo para los mayas, con el mes Poop y entonces ese día "iban limpios y galanos de sus unturas coloradas, y quitado el tizne negro de que andaban untados cuando ayunaban" (ibid.: 89). Entre los nahuas el tiznado a veces se aplicaba con el fin de evitar la salida del "calor" del cuerpo o del tonalli (López Austin, op. cit.: 91), y quizá los mayas también tomaban esta medida en esos días peligrosos con el mismo objetivo, Lacaso porque el tizne es un derivado del fuego-calor, o a manera de capa o coraza de protección que evitaba la introducción de seres malignos capaces de robar el "calor" del individuo?

Al terminar los días aciagos se prendía el fuego del año nuevo (J. y S. Nah, 1981: 19; Pérez, 1969: 29) con los rituales y ofrendas necesarias para que los dioses refrendaran el "calor" del nuevo período y con ello finalizaban los días álgidos en los que posiblemente se podía perder el "calor" de la persona, necesario para poder vivir y desarrollarse.

La búsqueda ávida del "calor" de los seres del cosmos se manifiesta en el Ritual de los Bacabes, después de una batalla campal que libra el terapeuta contra los males personificados que causan la enfermedad en el paciente, pues éste lleva a cabo atractivos intercambios "energéticos" para convencerlos de soltar a su presa. Dentro de las transacciones se encuentra, por supuesto, la ofrenda de sangre, contenedora de la esencia del hombre $(o o l)$, además de la "energía" albergada en las plantas medicinales, para con ello posiblemente darle el kinam, o gran "calor" solar que busca la entidad maligna: ${ }^{13}$ pues en este documento, dice el terapeuta: "¿Qué fue lo que tomé para hacer el cambio? Fue la yerba bacalché roja" (Ritual de los Bacabes op. cit.: 435). Es decir, lo que se intercambió para que saliera el mal del cuerpo del paciente y dejara de robarle su "energía",

${ }^{12}$ Entre los nahuas, las personas que necesitaban mantener un gran vigor en su tonalli o iban a pasar por situaciones de peligro, no podían cortarse ni lavarse el cabello (López Austin, op. cit.: 143 y 155).

${ }^{13} \mathrm{Ool}$, 'corazón formal' (Ciudad Real, op. cit. 1.: 598), o no corpóreo, centro del hombre o esencia. Posiblemente se encontraba estrechamente vinculado con el propio "calor" solar o kinam en el pensamiento maya, por lo siguiente: El corazón material (puczical), las entrañas en general y la sangre forman una importante unidad denominada olom. Olom tiene su raíz en el mencionado ool, "centro" o esencia del hombre, como una fuerza vital de todo el cuerpo concentrada en las entrañas, mediante el cual el ser humano mantenía una íntima y permanente comunicación con los dioses. Pareciera como si fuera un cordón umbilical entre las deidades y los individuos, a través del cual ellas manifestaban su contento o descontento de acuerdo con el comportamiento de éstos, entre otras cosas, con el detrimento de su salud. De ahí que al individuo sano se le denominara toh ool, 'centro' recto, o lo contrario, ch'alic ool, 'centro' aprovechado o cobrado por las divinidades (ibid.: 256 y 723; Chávez, 2002: 33-35). Podría ser que esa comunicación se diera especialmente con el astro dador del vital calor, el sol, de acuerdo con la relación o asociación corporal que brinda el Chilam Balam de Maní y de Kaua: vientre-corazón-sol (Bricker y Miram, op. cit.: 145). 
fue la yerba bacalché, pues como se observará, las plantas se percibían más bien como señuelos, y no como elementos capaces de matar al ser maligno con sus componentes, ya que a ellos parecían más bien gustarles los vegetales medicinales. Por ejemplo, también en el Ritual, el curandero indicaba: "ansioso estaba él [el agente de la enfermedad] por desayunarse la tuna roja. Hace un momento tomó de desayuno, el chacah rojo [...] el copó rojo" (ibid.: 403).

La cercanía de las yerbas curativas con el sol se complementa con el origen celeste de las mismas, como don divino, pues en los conjuros el chamán señalaba que las plantas medicinales debían ser atrapadas en el centro del cielo (ibid.: 297). Había que bajar la planta de las alturas, que más bien parece significar que descendía su "fuerza" o poder al momento de cortarla. Debía hacerse cuando no hubiera corrientes de aire, pues el terapeuta recomendaba: "Su planta es el sacyab, bajadla de ahí, del centro del cielo cuando el viento esté en calma" (ibid.: 342).

Posiblemente, la sugerencia de extraer la yerba de la tierra cuando no hubiera viento era una medida de protección para que los vientos personificados o seres dañinos no pudieran robar su "energía", kinam, o poderoso "calor" solar, porque recordemos que a este tipo de seres parecían gustarles las plantas medicinales.

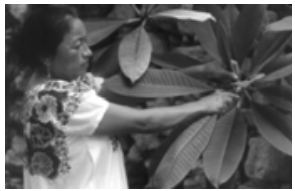

Las plantas medicinales contenían el poderoso "calor" o don solar (kinam) que ayudaba a aliviar las enfermedades del hombre. Rosa Pech, de Ekmul, Yucatán. Foto de Carlos Alcérreca

La capacidad del astro rey para otorgar sus beneficios a la humanidad se manifiesta en el aspecto del sol en forma del dios anciano, asociado con Itzamna, de los mayas prehispánicos del período Clásico, el más importante dentro del panteón maya yucateco. Los cackchiqueles de Panajachel lo invocaban antiguamente de manera diaria, al igual que los tzotziles actuales. Es el sol protector del hombre, capaz de alejar a los seres malévolos que atacan en la noche, y por eso le brindan oraciones para que les proporcione salud (Thompson, op. cit: 258 y 290-293). El sol es el padre, dador de vida, el calor está siempre presente entre ellos y en todo momento se le rinde culto (Gossen,1990: 59 y 60). 
Los Cantares de Dzitbalche contienen alabanzas especiales para el astro rey, como el cantar número seis, en donde se dice otro de los nombres de Itzamna: Hun Nab Ku, dios único ioh gran sol! (Libro de los Cantares de Dzitbalche, 1965: 46-47). En el número nueve se clama una oración por los dones otorgados por esta deidad que brinda salud, también creadora de todos los elementos del cosmos, como las plantas medicinales:

U uay- t'lumil Yan
u-dziic-tu-toh-yolal
h' tumen-u-yumil-
kaak-ha-iik-lum
yumil-uay-yokol cab
tut-tulah-c-bail
mentahan-tumen
yum-Hun-Naab-Ku
Latí-liti-Dziic-utz
Yeet-kaaz-ichil
Maloob-yetellob
Tu-men-lait-tie
Cu-dziic-u-zazilil
Yokol-cab-tumen
Laiti-u-yumil-tu
Lacal-baal

\author{
y que aquí en la tierra \\ da salud \\ porque es el Señor \\ del fuego, del agua, del aire, de la tierra. \\ Señor de este mundo \\ de todas las cosas \\ hechas por él \\ El señor Hun Nab Ku \\ es quien da lo bueno \\ y lo malo \\ entre los buenos y los malos. \\ Porque él \\ da su luz \\ sobre la tierra; porque \\ es el Dueño \\ de todas las cosas
}

(Libro de los Cantares de Dzitbalche, ibid.: 58). ${ }^{14}$

Pero no hay que olvidar la personalidad dual del sol, ya que también es capaz de enviar males terribles a la humanidad. Dentro de los padecimientos causados por él, registrados en el Chilam Balam de Kaua, se encuentra la pérdida del habla (nunil ik), provocada por su tiro (hul kin) y el dolor mordiente de corazón (chibal pucsikal lap), cuyos síntomas son el temor a los vecinos, a la sombra que se forma con sus rayos (sahac $u$ uinicil ti $u$ lakob ca ix $u$ boi uinic sahac ti tu men u kul kin) (Bricker y Miram, op. cit.: 353 y 371).

En el Ritual de los Bacabes se menciona frecuentemente la influencia del sol en la adquisición de la enfermedad, a manera de posesión del hombre por el dios, cuando le introduce males como el asma, el ronquido del habla pesada y el "Tancas del sol totalmente anaranjado" (Ritual de los Bacabes, op. cit.: 311 y 316317).

Kinam también es empleado en los documentos coloniales para manifestar el dolor, como kinam ool, 'dolor grande de cabeza' (Ciudad Real, op. cit.: 425), y en Yucatán, en la actualidad, es la forma de expresar un tipo de molestia muy fuer-

\footnotetext{
${ }^{14}$ Transcripción y traducción de Alfredo Barrera Vásquez.
} 
te con latidos llamado k'i'nan, como la aflicción intensa en la cabeza adquirida a partir de la exposición prolongada al sol, o la que se padece al momento del parto (Güémez, 2000: 317). En cuanto al empleo de este término para denominar a las plantas con cualidades "cálidas", en nuestros tiempos, médicos yucatecos y campechanos llaman kinal cuch, similar a la palabra kinam (fuerza, "calor" solar), así como choco cuch, a la carga "caliente" de las yerbas (García, et al., 1996: 122).

A continuación se presenta la lógica encontrada en la dosificación de los remedios medicinales y la frecuencia en su aplicación, así como las características cardinales de las plantas, aspectos también ligados con el sol, que confirman la importancia de este astro para la curación de las enfermedades.

\section{Colores cardinales y números rituales en las terapias curativas.}

Como recordaremos, los mayas percibían la tierra con cuatro orientaciones, reinadas cada una por una deidad (Landa, op. cit.: 62). Los cuatro puntos y el centro se relacionaban con los colores de acuerdo con el mito de la creación: chac, Este, rojo; sac, Norte, blanco; además de $e k$, Oeste, negro; kan, Sur, amarillo, y yaax, verde, centro. Cada uno de los dioses cardinales aportaban influencias benéficas y maléficas a la tierra, de acuerdo con el tiempo cíclico o repetitivo indígena y sus designios (Thompson, op. cit.: 242-244 y 336).

Esta base cardinal está presente, entre otros variados aspectos de la cultura maya, en la selección de las plantas medicinales, principalmente las que se encuentran o crecen al Oriente, región de salida del sol. Posiblemente en referencia al este de donde se ubica el terapeuta, o la persona que requiere curar sus males.

Las yerbas curativas marcadas con esta orientación en general, aunque no siempre, se prescriben para padecimientos que involucran la pérdida de sangre, probablemente porque las deidades causantes de las enfermedades rojas como la sangre y/o Lgraves? son los dioses del Oriente, o quizá porque al relacionar al Este con el sol, se le asocia con la sangre de la ofrenda. También podría ser posible que los dioses cardinales otorgaran los remedios para sanar los males que enviaban, dando como indicación el cuadrante del cosmos correspondiente para buscarlos.

Por ejemplo, para la orina, vómito y diarrea con sangre, al igual que para las hemorragias, se recomiendan plantas que crecen al Oriente (raíz de xeek tuak, kan thrix y de pomolche). Se indica la dirección a donde deberán ser colectadas (lakintan, Oriente), y frecuentemente se incluye dentro de los nombres de las mismas el color de la orientación (chac), como chac ac, chac sinkin, chac catzim, chac muc (J. y S. Nah, op. cit.: 32, 37, 39, 40, 43 y 47).

Otras direcciones se muestran, por ejemplo, para combatir diferentes tipos de pasmo, para los cuales se recomiendan las plantas que crecen en las direc- 
ciones correspondientes al Sur de color amarillo y al Norte blanco (ibid.: 32,37 , $39,40,43$ y 47). Es de notar que no se registran plantas con orientación al Poniente-negro, quizá por encontrase asociado este punto a la desaparición del sol, a lo oscuro, al inframundo, a la muerte y no a la salud.

En algunas ocasiones las plantas medicinales tienen características morfológicas rojas, como queriendo curar igual con igual. Por ejemplo, las puntas rojas de las hojas, lo rojizo del cuxum che, los rojos granos de la mazorca manchada, así como el chile colorado, entre otros, se sugieren para curar enfermedades con pérdida de sangre. En cuanto a otra indicación cromática se propone la incorporación de las yerbas que incluyen en sus nombres el término amarillo: como kan tun buk y kan mucuy che para enfermedades que provocan en el hombre una apariencia amarilla, como la ictericia (ibid: $32,33,36$ y 40).

Este tipo de asociaciones, de plantas de un color para enfermedades con características del mismo, no se encuentra en documentación del siglo xvı, pero sí hay una determinación de nombres de plantas medicinales de acuerdo con los colores de los puntos cardinales, como chacah, zac ppoppox, yaax ppoppox, kantunbub (Ciudad Real, op. cit. I: 669). también sin la inclusión de ek, negro Poniente.

La relación de los remedios y los órganos enfermos según los colores, manifiesta similitudes con la teoría de la signatura de Paracelso, de curar iguales con iguales, la cual forma parte de las ideas europeas de los conquistadores (Laín, 1972: 240). Creencia que no se puede descartar que pudiera haber sido aceptada fácilmente por los mayas por sus bases cosmogónicas, cardinales-cromáticas, aunque en los Chilam Balames, en los que se menciona este tipo de información, no se habla de la relación de los remedios y los órganos enfermos según las formas vegetales, como asientan las enseñanzas de Paracelso.

Al respecto de las yerbas curativas y los colores cardinales en tiempos más cercanos a la actualidad, Barrera Vásquez y Barrera Marín encontraron que dos quintas partes de las plantas estudiadas en la población de Cobá, en el siglo xx, incluían en los nombres mayas otorgados a las plantas los morfemas chak, sac, kan, yax y ek, al igual que se apunta en el Calepino de Motul del siglo xvı, mencionado líneas arriba. Chak y sac se indicaban con mayor frecuencia y el primero se registra con una ligera ventaja. Sac le podía otorgar a la planta el significado de imperfecta, falsa, imitadora de otra yerba, árbol o arbusto por compartir ciertas características comunes, y los cuatro colores podían calificar nombres genéricos, ${ }^{15}$ es decir, señalar un nivel de clasificación o agrupación de ciertas plantas con características iguales. Chac tenía el papel principal como clasificador y en segundo lugar le seguía sac (Barrera M. op. cit.: 474, 476, 478 y 483). Todo lo

${ }^{15}$ En la taxonomía actual, por ejemplo, hay varios tipos o clases de árboles balche', todos ellos dentro del género denominado en todo el mundo bajo la clasificación científica de Lonchocarpus, que comparten ciertas características comunes aquí y en otras partes del planeta, aunque reciban nombres en distintas lenguas. Algunos miembros de este género presentan ciertos elementos diferenciadores, a veces poco evidentes a simple vista, que se identifican por el segundo nombre, el de la especie: Lonchocarpus longistilus. 
anterior es una muestra de la continuidad y la importancia de los puntos cardinales en la percepción de las plantas en el pensamiento maya.

En la frecuencia y las dosis para medicar de los documentos coloniales, se muestran de manera constante los números $4,3,9$ y 13, principalmente los dos primeros, y es muy probable que tuvieran un sentido ritual o mágico, pues la divinización de los números era algo común entre los mayas. Para ellos el cuatro significaba las esquinas del mundo, u orientaciones cardinales, y también era la representación del anciano del cielo, quien era la fase solar benigna de Itzamna (Itzamna Kinich Ahau). El trece, por su parte, hacía referencia a las capas del cielo y el nueve a las del inframundo, con sus respectivas deidades, a las que había que implorar compasión para que dejaran de enviar males al individuo. Por esta importancia numeral es que la ceremonia del hetzmek entre los mayas actuales se lleva a cabo cuando un niño tiene 4 meses y de una niña se realiza a los 3 , porque son cuatro los lados de la milpa y tres los de las piedras que formarán el hogar en donde pasará el mayor tiempo la mujer. Igualmente en la ceremonia del Chaa chak, de la lluvia, realizada hasta nuestros días, los elementos que se incorporan se relacionan con estos números (Thompson, op. cit.: $210,239,243,266$ y 290-293).

En el Ritual de los Bacabes también hay una constante invocación a las orientaciones del mundo en donde se encuentran los cuatro dioses cardinales, de ahí el nombre del documento, y como es usual entre los mayas, se clama principalmente al del Oriente, lugar de salida del sol.

\section{Consideraciones finales}

La cultura maya se ha caracterizado por la plasticidad con la que se ha manejado a través del tiempo, al amoldarse a numerosos cambios, al adoptar, o más bien adaptar a su cultura nuevas creencias, manteniendo mucho de su esencia. Los documentos coloniales en maya son una muestra de ello, de ahí que a pesar de las abundantes referencias sobre la influencia de astros y planetas en el hombre, bajo la concepción europea, que se encuentran en ellos, sobresalga el sol, aunque sea de manera velada, en el profundo significado de los términos en lengua indígena mostrados de manera constante en los manuscritos.

El "frío" o el "calor" de las enfermedades combatidos por el "calor", fuerza o vitalidad del astro rey (kinam), tiene parecido con la forma de aliviar este tipo de padecimientos por los europeos, pero la diferencia esencial entre la percepción maya y la extranjera es la religiosa pagana, difícilmente explicada de manera clara en textos que los indígenas sabían que podían llegar a manos de los conquistadores cristianos, y ocasionarles problemas a quienes los escribieran. Las escasas ejemplificaciones de plantas que se especifican como "frías" para aliviar padecimientos "calientes" en la documentación maya colonial, bien podrían significar que en algunas ocasiones se concebían yerbas con estas cualida- 
des, como capaces de aliviar a sus contrarios; pero también podrían manifestar los atisbos de asimilación indígena del concepto del combate de enfermedades "frías-calientes" con sus opuestos, traída por los españoles. Pensamiento dicotómico que con el tiempo fue ganándole terreno al don de "calor" solar único, para establecerse de manera exitosa, tanto en la península de Yucatán, como en muchas partes del continente americano.

A pesar de que contamos con información parcial sobre el concepto solar de los tratamientos medicinales coloniales, el obsequio del astro rey (kinam) se muestra como fundamental en la terapéutica indígena, y en la manifestación de la enfermedad y la salud de los seres humanos. Como se habrá observado, el "calor" solar desataba en el pensamiento maya toda una dinámica, en la cual el hombre común recibía este regalo de manera cotidiana para que óptimamente se almacenara a lo largo de la vida en su persona, y con ello alcanzara perfección y sabiduría al llegar a la vejez. Como si al incrementar el don de la deidad máxima se acercara más a ella, y adquiriera también sus poderosas propiedades duales, benignas y malignas a su vez. Pero llegar a esa perfección era difícil, pues el ser humano debía luchar de manera permanente para mantenerlo en equilibrio y conservarlo, ya que este "calor" ocasionaba la eterna lucha entre vivos y muertos por el don de la fuerza vital, manzana de la discordia que movía al universo al pasar de un ser a otro en su cadena de persecución, aunque sólo los vivos tuvieran el privilegio de adquirirla de manera directa. Las deidades también entraban en el movimiento de este círculo de "energía", porque podían quitar el "calor" otorgado al hombre al enviarle males "fríos" como castigo por su mal comportamiento.

La importancia del sol, que se manifiesta en el cosmos cardinal indígena como consecuencia de su propio movimiento percibido desde la tierra, fungió como eje del desarrollo del pensamiento maya en sus múltiples esferas, y sigue manifestándose persistentemente en muchas comunidades indígenas actuales. Aun cuando el "calor" de los remedios ya no sea evocado como don sagrado del Sol.

Sin la venia de esta estrella se pensaba que las tinieblas acabarían con la vida en la tierra, y eso es una conclusión verídica también en nuestros tiempos bajo las premisas científicas, pues "el sol es el origen de casi toda la energía que sustenta la vida, la fuente del estado del tiempo, el árbitro de nuestro clima y nuestra conexión más cercana con los procesos que dan energía al cosmos" (Suplee, 2004:13). Así pues, no es de asombrar que la base de la supervivencia del cosmos y su orden fuera fincado por los mayas en la irradiación del astro rey, en la esperanza de su salida diaria del Oriente como representación de suma importancia del creador de todas las cosas. 


\section{BIBLIOGRAFIA}

Aveni, Anthony

1993 Observadores del cielo en el México antiguo. México: FCE.

Barrera Marín, Alfredo, Alfredo Barrera Vásquez y Rosa M. López Franco

1976 Nomenclatura etnobotánica maya. México: INAH, SEP.

Barrera Vásquez, Alfredo et al.

1995 Diccionario maya, $3^{3}$. ed. México: Porrúa.

Bocabulario de Maya Than

1993 René Acuña (ed.). México: UNAM, IIFL, Centro de Estudios Mayas (Fuentes para el Estudio de la Cultura Maya, 10).

Bricker, Victoria y Helga Miram

2002 An Encounter of Two Worlds. The Book of Chilam Balam of Kaua. Nueva Orleáns: Tulane University (Middle American Research Institute, 68).

Ciudad Real, Antonio

1995 Calepino de Motul, Ramón Arzápalo Marín (ed.). México: UNAM, IIA, 3 vols.

Chávez, Mónica

2002 "Medicina en la cosmovisión maya colonial. Terapias curativas en tres fuentes yucatecas del siglo XVI". Tesis de maestría en Etnohistoria. Mérida, Yucatán: Universidad Autónoma de Yucatán.

Chilam Balam de Ixil

1935 Comentarios de Alfredo Barrera Vásquez. Manuscrito de la Subdirección de Documentación: Colección de Códices, 63 ff. México: Biblioteca Nacional de Antropología e Historia.

Colby, Benjamín y Lore Colby

1986 El contador de los días, vida y discurso de un adivino ixil. México: FCE.

Foster, M. George

1994 Hippocrates Latin American Legacy. Humoral Medicine in the New World. Amsterdam: OPA, Gordon and Bleach, Science Publishers.

García, Hernán, Antonio Sierra y Gilberto Balám

1996 Medicina maya tradicional, confrontación con el sistema conceptual chino. México: EDUCE, FAM, VEMMESS, PRODUSSEP y Huelsz.

García, Luis "Galeno"

1972 Historia universal de la medicina, t. Il. España: Salvat. 
Gossen, Gary

1990 Los chamulas en el mundo del sol. México: Instituto Nacional para la Cultura y las Artes.

Guiémez, Miguel

2000 "La concepción del cuerpo humano, la maternidad y el dolor entre mujeres mayas yucatecas", Mesoamérica 21(39): 305-332. Vermont, EUA: Plumsock Mesoamerican Studies-CIRMA.

Guiteras, Calixta

1965 Los peligros del alma. Visión del mundo de un tzotzil. México: FCE.

Laín Entralgo, Pedro

1972 "La medicina hipocrática", Historia universal de la medicina, pp. 73-208. México: Archivo Salvat.

Landa, Diego de

1986 Relación de las cosas de Yucatán, pról. Ricardo Garibay. México: Porrúa.

Libro de los Cantares de Dzitbalche

1965 Trad. e introd. Alfredo Barrera Marín. México: INAH (Serie Investigaciones, 9).

López Austin, Alfredo

1996 Cuerpo humano e ideología. Las concepciones de los antiguos nahuas. México: UNAM, 2 ts.

Nah, José María y Secundino Nah

1981 Manuscritos de Tekax y Nah, trad. y com. Juan Ramón Bastarrachea. William Brito, Crescencio Aguayo Rodríguez, Domingo Dzul, Daniel Medina, Crescencio González, Joaquín Medina y Ermilo Yah (eds.). México: Grupo Dzibil.

Ossado, Ricardo

1983 El libro del judío, Alfredo Barrera Marín y Alfredo Barrera Vásquez (eds.). Xalapa, Veracruz: Instituto Nacional de Investigaciones sobre Recursos Bióticos.

1992 "Medicina doméstica. Descripción de los nombres y virtudes de las yerbas indígenas de Yucatán y las enfermedades a que se aplican", Las plantas medicinales de México, 6a. ed., pp. 553-606, Maximino Martínez (ed.). México: Ediciones Botas.

Pérez, Juan Pío

1949 Códice Pérez, trad. y com. Ermilo Solis. Mérida, Yucatán: Imprenta Oriente.

Pitarch, Pedro

1996 Ch'ulel: una etnografia de las almas tzeltales. México: FCE.

Redfield, Robert y Alfonso Villa Rojas

1964 Chan Kom, a Maya Village, $2^{\text {a }}$ ed. Chicago: The University of Chicago Press. 
Relaciones histórico-geográficas de la Gobernación de Yucatán

1983 Mercedes de la Garza, Ana Luisa Izquierdo y M. del Carmen León (eds.). México: UNAM, IIFL, Centro de Estudios Mayas (Fuentes para el Estudio de la Cultura Maya, 1) 2 vols.

Ritual de los Bacabes

1987 Ramón Arzápalo Marín (ed.). México: UNAM, IIFL, Centro de Estudios Mayas (Fuentes para el Estudio de la Cultura Maya, 5).

Sosa, Victoria, Salvador Flores, Víctor Rico, Rafael Lira y Javier Ortiz

1985 Etnoflora yucatanense. Lista florística y sinonimia maya. Xalapa, Veracruz. México: Instituto Nacional de Investigaciones sobre Recursos Bióticos, fascículo1.

Suplee, Curt

2004 "El Sol", National Geographic en español, julio, 15 (1): 6-33. México: National Geographic Society, Editorial Televisa.

Thompson, Eric

1975 Historia y religión de los mayas. México: Siglo XXI.

1993 Un comentario al Códice Dresde, libro de jeroglíficos mayas. México: FCE.

Villa Rojas, Alfonso

1981 "Terapéutica tradicional y medicina moderna entre los mayas de Yucatán", Anales de Antropología. México: UNAM. 